\title{
Molecular survey of piroplasm species from selected areas of China and Pakistan
}

\author{
Muhammad Adeel Hassan', Junlong Liu', Muhammad Rashid', Naveed Iqbal', Guiquan Guan', Hong Yin ${ }^{1,2}$ \\ and Jianxun Luo ${ }^{1 *}$
}

\begin{abstract}
Background: Piroplasmosis is an important animal disease that is a major constraint to the development of the livestock industry, often resulting in significant economic losses. Therefore, there is an urgent need to further understand the etiology of this and other tick-borne infections.

Methods: Blood samples were collected from tick-infested animals from the Chakwal, Jhang, and Faisalabad districts of Punjab, Pakistan and from peri-urban areas around Hohhot, Inner Mongolia, China to investigate the presence of Babesia and Theileria species. In total, 450 blood samples were collected with FTA cards from cattle of the study areas of Pakistan; the genomic (g)DNA of one hundred and twenty samples from cattle in Inner Mongolia were provided by the Lanzhou Veterinary Research Institute, China. Following the extraction of gDNA, the 185 rRNA gene (V4 hypervariable region) of piroplasms was amplified in all samples using semi-nested PCR. Positively identified samples were sequenced for the identification of Theileria and Babesia species. The partial full-length sequence of 185 rDNA was amplified for species confirmation of Theileria-positive samples, whereas the RAP-1c gene was amplified for Babesia bigemina-positive samples.
\end{abstract}

Results: Semi-nested PCR results revealed that 144 (25.26\%) samples were positive for piroplasms. Theileria annulata was the most prevalent species (115/144; 20.17\%), followed by Theileria orientalis (16/144; 2.80\%). Among Babesia, the only species recorded was Babesia bigemina (13/144; 2.28\%).

Conclusion: The present study reveals new data on the prevalence of piroplasm species in bovine populations of selected areas of China and Pakistan and their phylogenetic relationships. It is also the first detailed report of T. orientalis from native animals in Pakistan.

Keywords: Theileria, Babesia, PCR, China, Pakistan

\section{Background}

The livestock industry is an important part of the economy in Pakistan, contributing $11.6 \%$ of the total gross domestic product during the economic year 2016-2017. According to statistics, there are 82.1 million heads of cattle and buffalo in Pakistan [1]. Although it is estimated that two thirds of the human population of Pakistan is involved in the livestock sector either directly or indirectly, management practices and disease prevention and control strategies are poorly developed.

\footnotetext{
* Correspondence: luojianxun@caas.cn

${ }^{1}$ State Key Laboratory of Veterinary Etiological Biology, Key Laboratory of Veterinary Parasitology of Gansu Province, Lanzhou Veterinary Research Institute, Chinese Academy of Agricultural Sciences, Xujiaping 1, Lanzhou, Gansu 730046, People's Republic of China

Full list of author information is available at the end of the article
}

Piroplasmosis is one of the most important diseases, constraining the livestock industry in tropical and subtropical areas of the world [2]. It is caused by species of two genera of haemoprotozoa, Theileria and Babesia. In general, Theileria annulata and Theileria parva are considered to be the most pathogenic species, causing theileriosis in cattle and buffalo populations of tropical and subtropical areas, whereas Theileria mutans and Theileria orientalis are responsible for asymptomatic disease [3]. Hard ticks of the genera Hyalomma, Rhipicephalus, Haemaphysalis and Amblyomma are vectors of these Theileria spp., resulting in fatal economic losses in endemic areas of Asia and Africa [4,5]. Clinical manifestations of theileriosis include: increased body temperature $\left(40-41.5{ }^{\circ} \mathrm{C}\right)$, followed by depression, watery secretion

(C) The Author(s). 2018 Open Access This article is distributed under the terms of the Creative Commons Attribution 4.0 International License (http://creativecommons.org/licenses/by/4.0/), which permits unrestricted use, distribution, and 
from the eyes, nasal discharge, inflammation of the lymph nodes and anemia [6].

Bovine babesiosis is mainly caused by haemoprotozoans of the genus Babesia, including Babesia bovis, Babesia divergens, Babesia ovata, Babesia major and Babesia bigemina. Among these, B. bovis and B. bigemina are the most pathogenic species, transmitted by Rhipicephalus microplus, which is a globally distributed tick species. The other species, B. ovata and B. major, are transmitted by Haemaphysalis longicornis and Haemaphysalis punctata, respectively [7]. The clinical signs of babesiosis include fever, icterus, hemoglobinuria and anemia [8].

Diagnosis of the piroplasm species is usually done using Giemsa's-stained blood smears, but this method cannot be utilized to identify carriers of the parasites, because the levels of parasitemia are low [9]. Studies show that carrier animals can be source of infection for a long time [10]. Serological assays have also been developed for detecting these parasite infections. Compared with other serological assays, such as indirect fluorescent antibody tests and compliment fixation tests, enzyme-linked immunosorbent assays are highly sensitive and easy to standardize, and, thus, are a preferred method for epidemiological studies $[11,12]$. However, low specificity and false negative results in the case of carrier animals can hinder the efficacy of these tests [13]. However, for epidemiological surveys, it is recommended to detect carrier animals [14]. Molecular techniques can used comparatively reliably to determine the species spectrum of parasites in population studies, and species-specific PCR assays have been established for this purpose. Meanwhile, the development of reverse line blot (RLB) hybridization has enabled the simultaneous detection of various piroplasm species $[15,16]$. PCR has been proven to be more specific and sensitive method for the identification of carrier and diseased animals in various studies worldwide.

In Pakistan, most published studies report the use of Giemsa's staining for the detection of piroplasm species [5]. However, there have also been a few reports utilizing molecular techniques for diagnosis. For example, $T$. annulata has been detected by using species-specific primers in PCR $[8,17-19]$, but no confirmation of the results by DNA sequencing was provided. Gebrekidan et al. [20] reported the presence of T. orientalis in Pakistan for the first time from imported and native animals, reporting no $T$. orientalis in native animals by conventional PCR and low prevalence (6\%) in native cattle as compared with imported animals (25.4\%) using multiplexed tandem PCR. Recently, Hassan et al. [21, 22] determined the presence of Theileria spp. by using recombinase polymerase amplification. In terms of the detection of Babesia spp., PCR has been used in epidemiological studies reporting the presence of $B$. bovis and $B$. bigemina $[8,23,24]$. In the present study, semi-nested PCR was utilized to identify several species of piroplasm at the molecular level.

\section{Methods}

\section{Sample collection}

During July and August 2016, 450 blood samples were randomly collected from tick-infested but asymptomatic cattle in the Chakwal, Jhang and Faisalabad districts in Punjab, Pakistan. Blood was collected from the jugular vein using $10-\mathrm{ml}$ disposable syringes and transferred to the four circles of a Whatman ${ }^{\oplus}$ FTA card (GE Healthcare Limited, Buckinghamshire HP7 9NA, UK) and allowed to air-dry. The FTA cards were shipped to Lanzhou Veterinary Research Institute, Chinese Academy of Agricultural Sciences Lanzhou, China for further processing. In addition, 120 samples of genomic (g)DNA collected from the blood of cattle in the field from Inner Mongolia, China were provided by the Vectors and Vector Borne Diseases Laboratory, Lanzhou Veterinary Research Institute, China.

\section{DNA extraction}

Genomic DNA was extracted from the FTA cards using the genomic DNA purification kit (Qiagen, Hilden, Germany) according to the manufacturer's instructions. The DNA concentration was determined with a NanoDrop 2000 spectrophotometer (Nanodrop Technologies ${ }^{\ominus}$, Wilmington, DE, USA). DNA was stored at $-20{ }^{\circ} \mathrm{C}$ until further analysis.

Table 1 Details of the primers used in this study

\begin{tabular}{|c|c|c|c|c|}
\hline Primer Name & Sequence $\left(5^{\prime}-3^{\prime}\right)$ & Product size $(b p)$ & Annealing $T\left({ }^{\circ} \mathrm{C}\right)$ & Reference \\
\hline RLB-F2 & GACACAGGGAGGTAGTGACAAG & 393 & 52 & [25] \\
\hline RLB-R2 & CTAAGAATTTCACCTCTGACAGT & & & \\
\hline RLB-Fint & GACAAGAAATAACAATACRGGGC & & & \\
\hline NBab-1F & AAGCCATGCATGTCTAAGTAGAAGCTITT & $\sim 1600$ & 58 & [26] \\
\hline 18SRev-BT & GAATAATTCACCGGATCACTCG & & & \\
\hline B.birap-1cseq-F & TTACGCTGCTTACTACAGCTTCA & 1054 & 57 & [7] \\
\hline B.birap-1cRc & TTACGACGATCGTTGAAGTACTTC & & & \\
\hline
\end{tabular}


Table 2 Detection of piroplasm species from selected areas of China and Pakistan using semi-nested PCR

\begin{tabular}{llllll}
\hline Area & $\begin{array}{l}\text { T. annulata } \\
n(\%)\end{array}$ & $\begin{array}{l}\text { T. orientalis } \\
n(\%)\end{array}$ & $\begin{array}{l}\text { B. bigemina } \\
n(\%)\end{array}$ & $\begin{array}{l}\text { Negative } \\
n(\%)\end{array}$ \\
\hline Chakwal & $88(46.80)$ & $12(6.38)$ & $10(5.31)$ & $78(41.48)$ & 188 \\
Jhang & $5(2.96)$ & $0(0)$ & $0(0)$ & $1(97.04)$ & 169 \\
Faisalabad & $10(10.75)$ & $2(2.15)$ & $2(1.67)$ & $80(86.02)$ & $104(86.67)$ \\
Inner Mongolia & $12(10.00)$ & $2(1.67)$ & $13(2.28)$ & $426(74.73)$ & 120 \\
Total & $115(20.17)$ & $16(2.80)$ & & 570 \\
\hline
\end{tabular}

\section{PCR amplification}

Semi-nested PCR was used to amplify the V4 hypervariable region of the $18 S$ rRNA gene from the gDNA of cattle. For this purpose, universal primers were used that can amplify several Babesia and Theileria spp. [25]. The first PCR reaction was conducted using primers RLB-F2 and RLB-R2, as shown in Table 1 . The reactions were performed in a final volume of $25 \mu$ l containing $12.5 \mu \mathrm{l}$ Premix Taq DNA polymerase (TaKaRa, Beijing, China), $1.0 \mu \mathrm{M}$ of each primer, and $2 \mu \mathrm{l}$ of DNA template. The second PCR was carried out with the primer RLB-FINT (as shown in Table 2) used as the forward primer, together with RLB-R2. The reaction mixture was as in the first PCR reaction, except the template was replaced by $2 \mu \mathrm{l}$ of the first PCR product. PCR products were electrophoresed on a $1.5 \%$ agarose gel and visualized under UV light. Positive PCR products were excised from the gel and purified using a BIOMIGA EZgene ${ }^{\mathrm{TM}}$ Gel/PCR Extraction Kit (San Diego, CA USA). The DNA fragment was cloned into pGEM-T Easy Vector (Promega, Madison, USA). Escherichia coli Trans $5 \alpha$ (TaKaRa, China) was transformed and plasmid DNA from the selected clones was identified using PCR with the set of primers T7 (5'-TAA TAC GAC TCA CTA TAG GG-3') and SP6 (5'-ATT TAG GTG ACA CTA TAG-3') to verify the presence of correct inserts in selected clones before proceeding with the sequencing process. The reaction cycling in the second PCR was optimized with primer annealing at $54{ }^{\circ} \mathrm{C}$ for $30 \mathrm{~s}$. The correct inserted clones were shipped to Genescript ${ }^{\ominus}$ (Shanghai, China) for sequencing [26].

To confirm the Theileria spp., a long fragment of the $18 \mathrm{~S}$ rRNA gene $(\sim 1600 \mathrm{bp})$ was amplified from samples that were positive in short sequence amplification. The long fragment of the RAP-1c gene of B. bigemina (1054 bp) was amplified for species confirmation. The primers used are listed in Table 1.

\section{Phylogenetic analysis}

The obtained sequences were aligned using the MegAlign component of the DNAStar software program (Version 4.0 DNAStar, Madison, USA). After alignment with related Theileria and Babesia spp. $18 S$ rDNA sequences retrieved from GenBank, parts of the cloning vector region were removed manually. The resulting sequences were then submitted to the GenBank database. A phylogenetic tree was generated based on the cloned

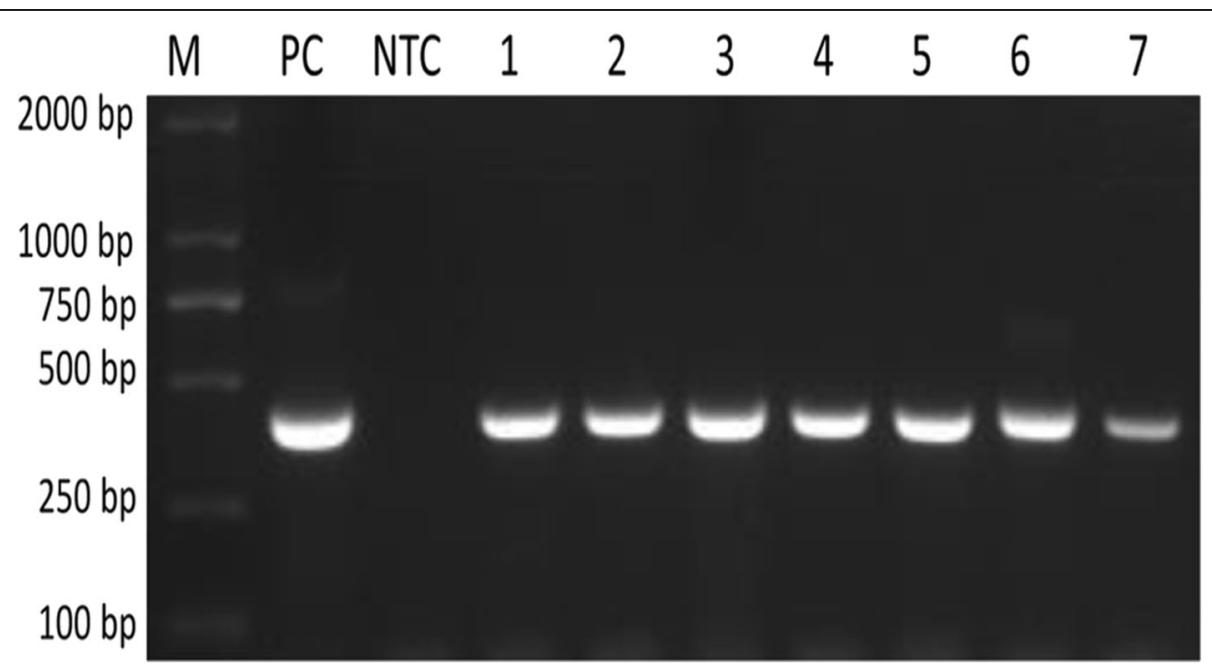

Fig. 1 1.5\% agarose gel electrophoresis image showing a 393 bp band for the amplification of the V4 region of the 185 rRNA gene of piroplasm species. Lane M: DL 2000 DNA marker; Lane PC: positive control; Lane NTC: negative test control; Lanes 1-7: positive identified samples by semi-nested PCR 
sequences and the related Theileria/Babesia $18 S$ rDNA sequences in GenBank by using the neighbor-joining algorithm in the MEGA 6.0 software [27]. The evolutionary distances were computed using the Kimura two-parameter method [28]. The phylogenetic trees based on long fragment of $18 \mathrm{~S}$ rRNA gene of Theileria spp. and $R A P-1 c$ gene of B. bigemina were generated using the above-mentioned procedure.

\section{Results}

Semi-nested PCR was used to detect Theileria and Babesia spp. by targeting the V4 region of the $18 S$ rRNA gene and amplifying a 393-bp fragment (Fig. 1). Of the 570 bovine blood samples, 144 (25.26\%) samples were positive for piroplasms, showing an approximately 393-bp product band in the agarose gel. The PCR products of all the positive detected 144 samples were sequenced. The phylogenetic analysis based on the short amplified sequences of the $18 \mathrm{~S}$ rDNA V4 hypervariable region revealed three piroplasm species (T. annulata, $T$. orientalis and B. bigemina) in samples from the two study areas (Fig. 2). Theileria annulata (115/570; 20.17\%) was the most prevalent piroplasm species. The highest prevalence of $T$. annulata was found in Chakwal (46.80\%), followed by Faisalabad (10.75\%), Inner Mongolia (10.0\%) and Jhang (2.96\%). The second most abundant species was $T$. orientalis $(16 / 570 ; 2.80 \%)$. The highest prevalence of $T$. orientalis was found in Chakwal (6.38\%), followed by Faisalabad (2.15\%) and Inner Mongolia (1.67\%). Theileria orientalis was not found in Jhang. Babesia bigemina (13/570; 2.28\%) was the only species of Babesia found in any of the samples. The highest prevalence of $B$. bigemina was in Chakwal (5.31\%), followed by Inner Mongolia (1.67\%) and Faisalabad (1.07\%); this species was not found in Jhang. The highest piroplasm overall prevalence was

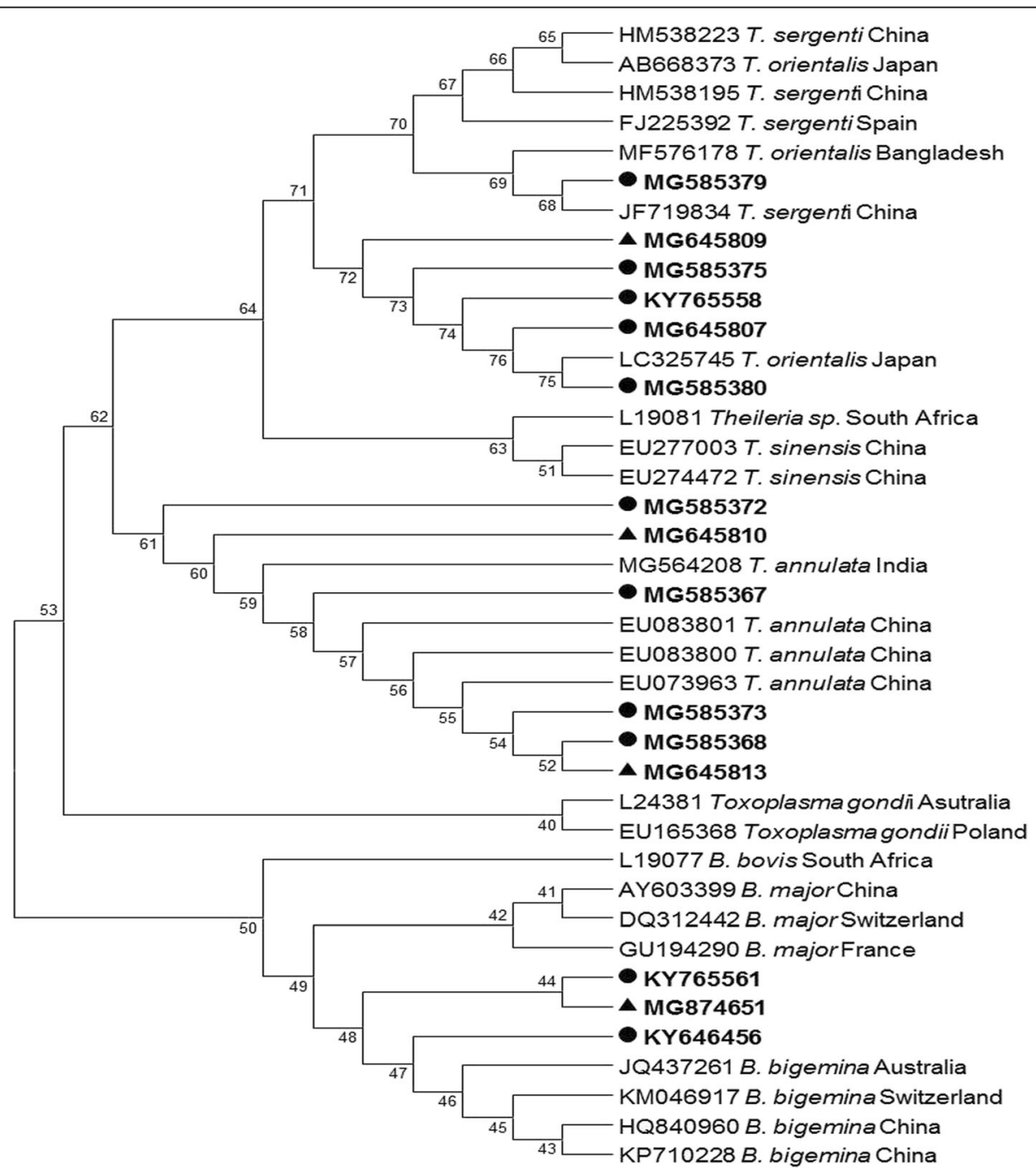

Fig. 2 Phylogenetic tree for Theileria spp. and Babesia spp. based on the V4 region of the 185 rRNA gene sequences. The parasites identified in the present study are marked in bold. Samples from Pakistan are indicated by circles; samples from China are indicated by triangles 
recorded in the samples from Chakwal (110/188; $58.51 \%)$, followed by Faisalabad (13/93; 13.97\%), Inner Mongolia $(16 / 120 ; 13.33 \%)$ and Jhang $(5 / 169 ; 2.95 \%)$ (Table 2). The results of the long fragment amplification confirmed the presence of these species. The phylogenetic tree revealed the relationships between these species.

\section{Discussion}

In Pakistan, small-holder cattle farming systems are common for both commercial and domestic purposes in resource-poor rural communities. In recent years, farmers have adopted commercial farming using modern techniques and imported breeds of cattle (Bos taurus) because of their higher milk yield potential. However, the imported animals are more prone to ticks and tick- borne diseases compared with the local breeds (Bos indicus), resulting in mortality rates as high as $80 \%$ in the former compared with $<20 \%$ in the latter animals. Among many tick-borne diseases, bovine theileriosis, predominantly caused by $T$. annulata, is considered among the most economically notorious diseases [5]. To date, most studies of bovine theileriosis have been conducted by using conventional microscopy, which, because of its low sensitivity and specificity, does not truly represent the actual distribution of the species involved. Recently, a study reported the prevalence of $T$. orientalis from imported and native bovine populations of Pakistan, describing $24.5 \%$ positive results in imported animals as compared with $6 \%$ positive results in native cattle [20]. In China, four Babesia species (B. bovis, B. bigemina, B. major and $B$. ovata) have been described as being responsible for cattle, buffalo and yak babesiosis [29, 30]. Recently, Liu et al. [30]

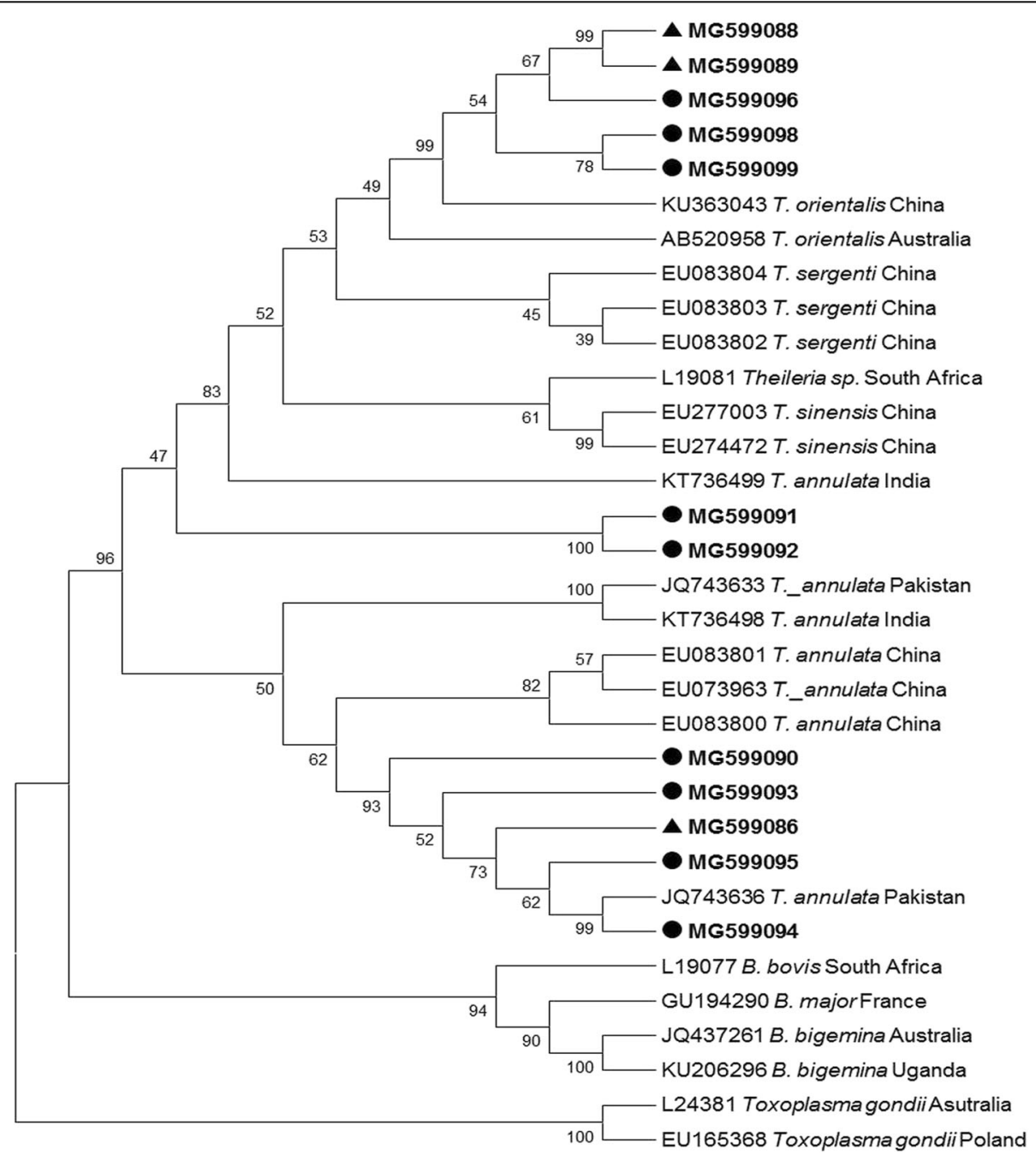

Fig. 3 Phylogenetic tree for Theileria spp. constructed based on the 185 rRNA gene sequences. The parasites identified in the present study are marked in bold. Samples from Pakistan are indicated by circles; samples from China are indicated by triangles 


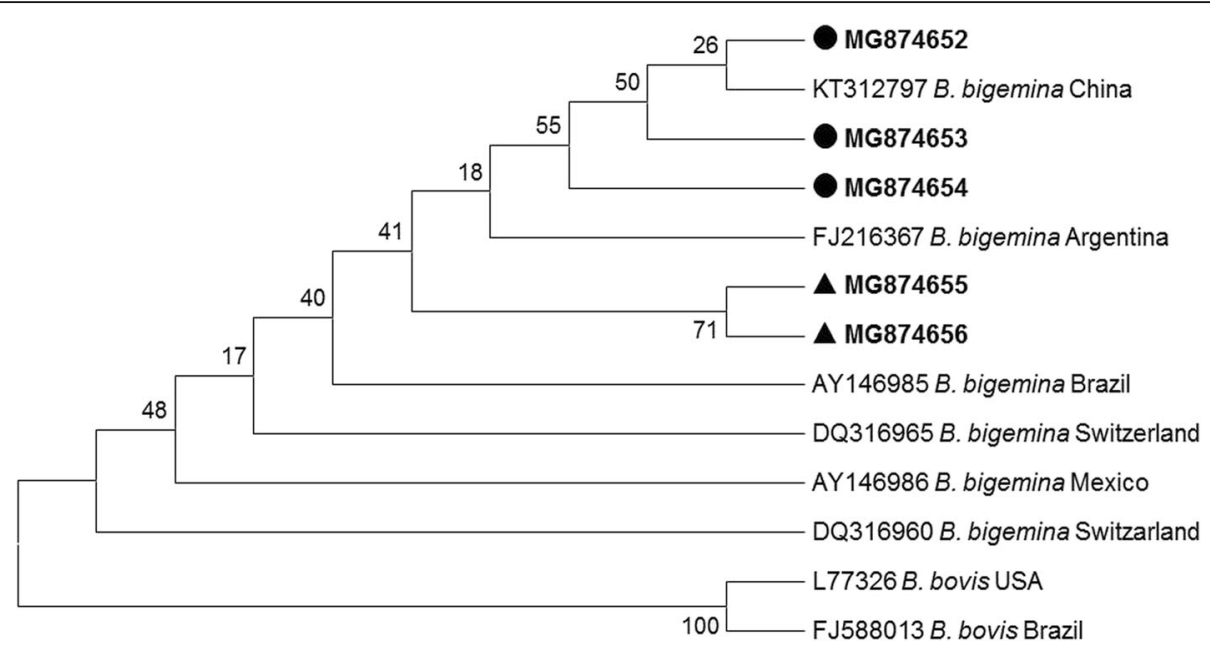

Fig. 4 Phylogenetic tree for Babesia bigemina constructed based on the rhoptry associated protein (RAP-1C) gene sequences. The sequences identified in the present study are marked in bold. Samples from Pakistan are indicated by circles; samples from China are indicated by triangles

detected a new bovine Babesia, similar to $B$. venatorum and Qin et al. [31] reported an epidemiological study of B. orientalis in China. Among different members of Theileria, T. annulata, T. orientalis and T. sinensis, have been reported to cause the disease in China [4]. Recently, T. luwenshuni was reported for the first time in yaks [32].

In the present study, phylogenetic analysis of $T$. annulata short sequences showed maximum similarity with sequences already reported from India and China, whereas, on the basis of long fragment amplified sequences, most of the $T$. annulata sequences were found to be closely related to sequences already reported from Pakistan [17] and Iran (GenBank: KF429799). A few short sequences of $T$. orientalis showed similarity with sequences already reported from China, whereas others showed a close relationship with sequences from Japan. Some of the long $T$. orientalis sequences reported from Pakistan were closely related to sequences already reported from China (GenBank: KU363043) and Pakistan (GenBank:JQ743636), whereas a few sequences were not closely related to sequences in GenBank and appear to be distinct (GenBank: MG599098 and MG599099) (Fig. 3). The sequence JQ743636 was obtained from gDNA of cattle imported from Australia to Pakistan. This shows that the disease in native animals might have come from infected imported animals. The $B$. bigemina short sequences were analyzed by using other reference sequences of the same gene [7]. These showed a relationship with reported sequences from Australia, China and Switzerland (Fig. 2). The long fragment sequences revealed a relationship with sequences reported from China and Argentina, with a few sequences occupying a distinct position in the phylogenetic tree (Fig. 4).

\section{Conclusions}

The present study reports the simultaneous detection of multiple piroplasm species, and the genetic relationship between them, from selected areas of Pakistan and China. The results of this study could be helpful for providing a basis for vaccine production and control strategies for these economically important piroplasmosis.

\section{Acknowledgements}

The authors acknowledge International Science Editing to improve the English of the manuscript.

\section{Funding}

This study was financially supported by the National Key R\&D Program of China (2017YFD0501200, 2016YFC1202000); 973 Program (2015CB150300); NSFC (No:31402189, No:31372432); ASTIP, FRIP (2014ZL010), CAAS; NBCIS (CARS-38); Jiangsu Co-Innovation Center for the Prevention and Control of Important Animal Infectious Disease and Zoonose, and State Key Laboratory of Veterinary Etiological Biology Projects.

\section{Availability of data and materials}

The reference sequences of the present study were submitted to the GenBank database under the accession numbers as follows: T. annulata (KY626168, MF427698, MG585358-73, MG599086-87, MG599090-95, MG645810-13); T. orientalis (KY765557-58, MG585374-83, MG599088-89, MG599096-99); B. bigemina (KY646456, KY765561-62, MG874651-56).

\section{Authors' contributions}

$J \mathrm{~L}, \mathrm{HY}$ and $\mathrm{GG}$ planned the research. MAH and $\mathrm{NI}$ collected the samples. MAH, $M R$ and $J \mathrm{~L}$ conducted the experiments. MAH drafted the manuscript. JL and GG revised the manuscript. All authors read and approved the final manuscript.

\section{Ethics approval}

The present study was approved by the Animal Ethics Committee of Lanzhou Veterinary Research Institute, CAAS (No. LVRIAEC 2013-010). The procedures for acquiring the field samples were approved by the Animal Welfare Ethics defined by University of Agriculture Faisalabad, Pakistan.

\section{Consent for publication}

Not applicable.

Competing interests

The authors declare that they have no competing interests. 


\section{Publisher's Note}

Springer Nature remains neutral with regard to jurisdictional claims in published maps and institutional affiliations.

\section{Author details \\ 'State Key Laboratory of Veterinary Etiological Biology, Key Laboratory of Veterinary Parasitology of Gansu Province, Lanzhou Veterinary Research Institute, Chinese Academy of Agricultural Sciences, Xujiaping 1, Lanzhou, Gansu 730046, People's Republic of China. ${ }^{2}$ Jiangsu Co-Innovation Center for the Prevention and Control of Important Animal Infectious Disease and} Zoonose, Yangzhou University, Yangzhou 225009, People's Republic of China.

Received: 23 May 2018 Accepted: 24 July 2018

Published online: 07 August 2018

\section{References}

1. Anonymous. Economic Survey of Pakistan. 2016-2017. 2017. http://www. finance.gov.pk/survey_1617.html

2. Sivakumar T, Hayashida K, Sugimoto C, Yokoyama N. Evolution and genetic diversity of Theileria. Infect Genet Evol. 2014;27:250-63.

3. Uilenberg G, Mpangala C, McGregor W, Callow L. Biological differences between African Theileria mutans (Theiler, 1906) and two benign species of Theileria of cattle in Australia and Britain. Aust Vet J. 1977:53:271-3.

4. Abdallah MO, Niu Q, Yang J, Hassan MA, Yu P, Guan G, et al. Identification of 12 piroplasms infecting ten tick species in China using reverse line blot hybridization. J Parasitol. 2017;103:221-7.

5. Jabbar A, Abbas T, Saddiqi HA, Qamar MF, Gasser RB. Tick-borne diseases of bovines in Pakistan: major scope for future research and improved control. Parasit Vectors. 2015;8:283

6. Gubbels J, Katzer F, Hide G, Jongejan F, Shiels BR. Generation of a mosaic pattern of diversity in the major merozoite-piroplasm surface antigen of Theileria annulata. Mol Biochem Parasitol. 2000;110:23-32.

7. Niu Q, Liu Z, Yu P, Yang J, Abdallah MO, Guan G, et al. Genetic characterization and molecular survey of Babesia bovis, Babesia bigemina and Babesia ovata in cattle, dairy cattle and yaks in China. Parasit Vectors. 2015;8:518.

8. Durrani A, Kamal N. Identification of ticks and detection of blood protozoa in Friesian cattle by polymerase chain reaction test and estimation of blood parameters in district Kasur Pakistan. Trop Anim Health Prod. 2008:40:441-7.

9. Shayan P, Rahbari S. Simultaneous differentiation between Theileria spp. and Babesia spp. on stained blood smear using PCR. Parasitol Res. 2005:97:281-6.

10. Oliveira-Sequeira T, Oliveira M, Araujo J, Amarante A. PCR-based detection of Babesia bovis and Babesia bigemina in their natural host Boophilus microplus and cattle. Int J Parasitol. 2005:35:105-11.

11. Zhao S, Liu J, Zhao H, Li Y, Xie J, Liu A, et al. Evaluating an indirect rMPSP enzyme-linked immunosorbent assay for the detection of bovine Theileria infection in China. Parasitol Res. 2017;116:667-76.

12. Tian Z, Du X, Du J, Gao S, Yu R, Hassan MA, et al. Development of an indirect ELISA based on Spm2 protein for detection of tropical theileriosis. Acta Trop. 2018;182:232-6.

13. Passos LMF, Bell-Sakyi L, Brown CGD. Immunochemical characterization of in vitro culture-derived antigens of Babesia bovis and Babesia bigemina. Vet Parasitol. 1998;76:239-49.

14. Fahrimal Y, Goff W, Jasmer DP. Detection of Babesia bovis carrier cattle by using polymerase chain reaction amplification of parasite DNA. J Clin Microbiol. 1992:30:1374-9.

15. Iqbal F, Khattak R, Ozubek S, Khattak M, Rasul A, Aktas M. Application of the reverse line blot assay for the molecular detection of Theileria and Babesia sp. in sheep and goat blood samples from Pakistan. Iran J Parasitol. 2013;8:289.

16. M'ghirbi Y, Hurtado A, Brandika J, Khlif K, Ketata Z, Bouattour A. A molecular survey of Theileria and Babesia parasites in cattle, with a note on the distribution of ticks in Tunisia. Parasitol Res. 2008;103:435-42.

17. Khan MK, He L, Hussain A, Azam S, Zhang WJ, Wang LX, et al. Molecular epidemiology of Theileria annulata and identification of 18S rRNA gene and ITS regions sequences variants in apparently healthy buffaloes and cattle in Pakistan. Infect Genet Evol. 2013;13:124-32.

18. Khattak R, Rabib M, Khan Z, Ishaq M, Hameed H, Taqddus A, et al. A comparison of two different techniques for the detection of blood parasite, Theileria annulata, in cattle from two districts in Khyber Pukhtoon Khwa Province (Pakistan). Parasite. 2012;19:91-5.
19. Shahnawaz S, Ali M, Aslam M, Fatima R, Chaudhry Z, Hassan M, et al. A study on the prevalence of a tick-transmitted pathogen, Theileria annulata, and hematological profile of cattle from southern Punjab (Pakistan). Parasitol Res. 2011;109:1155-60.

20. Gebrekidan H, Abbas T, Wajid M, Ali A, Gasser RB, Jabbar A. Molecular characterization of Theileria orientalis in imported and native bovines from Pakistan. Infect Genet Evol. 2017:47:19-25.

21. Hassan MA, Liu J, Sajid MS, Rashid M, Mahmood A, Abbas Q, et al. Simultaneous detection of Theileria annulata and Theileria orientalis using recombinase polymerase amplification. Ticks Tick Borne Dis. 2018;9:1002-5

22. Hassan MA, Liu J, Sajid MS, Mahmood A, Zhao S, Abbas Q, et al. Molecular detection of Theileria annulata in cattle from different regions of Punjab, Pakistan, by using recombinase polymerase amplification and polymerase chain reaction. J Parasitol. 2018;104:196-201.

23. Chaudhry Z, Suleman M, Younus M, Aslim A. Molecular detection of Babesia bigemina and Babesia bovis in crossbred carrier cattle through PCR. Pak J Zool. 2010:42:201-4.

24. Zulfiqar S, Shahnawaz S, Ali M, Bhutta AM, Iqbal S, Hayat S, et al. Detection of Babesia bovis in blood samples and its effect on the hematological and serum biochemical profile in large ruminants from southern Punjab. Asian Pac J Trop Biomed. 2012;2:104-8.

25. Gubbels J, De Vos A, Van der Weide M, Viseras J, Schouls L, De Vries E, et al. Simultaneous detection of bovine Theileria and Babesia species by reverse line blot hybridization. J Clin Microbiol. 1999:37:1782-9.

26. Liu J, Yang J, Guan G, Liu A, Wang B, Luo J, et al. Molecular detection and identification of piroplasms in sika deer (Cervus nippon) from Jilin Province China. Parasit Vectors. 2016;9:156

27. Tamura K, Stecher G, Peterson D, Filipski A, Kumar S. MEGA6: Molecular Evolutionary Genetics Analysis version 6.0. Mol Biol Evol. 2013;30:2725-9.

28. Kimura M. A simple method for estimating evolutionary rates of base substitutions through comparative studies of nucleotide sequences. J Mol Evol. 1980;16:111-20

29. Luo J, Yin H, Liu Z, Yang D, Guan G, Liu A, et al. Molecular phylogenetic studies on an unnamed bovine Babesia sp. based on small subunit ribosomal RNA gene sequences. Vet Parasitol. 2005;133:1-6.

30. Liu J, Guan G, Li Y, Liu A, Luo J, Yin H. A molecular survey of Babesia species and detection of a new Babesia species by DNA related to $B$. venatorum from white yaks in Tianzhu, China. Front Microbiol. 2017:8:419.

31. Qin SY, Wang JL, Ning HR, Tan QD, Yin MY, Zhang XX, et al. First report of Babesia bigemina infection in white yaks in China. Acta Trop. 2015;145:52-4.

32. Qin G, Li Y, Liu J, Liu Z, Yang J, Zhang L, et al. Molecular detection and characterization of Theileria infection in cattle and yaks from Tibet Plateau Region China. Parasitol Res. 2016;115:2647-52.

Ready to submit your research? Choose BMC and benefit from:

- fast, convenient online submission

- thorough peer review by experienced researchers in your field

- rapid publication on acceptance

- support for research data, including large and complex data types

- gold Open Access which fosters wider collaboration and increased citations

- maximum visibility for your research: over $100 \mathrm{M}$ website views per year

At BMC, research is always in progress.

Learn more biomedcentral.com/submissions 